Article

\title{
Two New Jaspamide Derivatives from the Marine Sponge Jaspis splendens
}

Sherif S. Ebada ${ }^{1,6}$, Victor Wray ${ }^{2}$, Nicole J. de Voogd ${ }^{3}$, Zhiwei Deng ${ }^{4}$, Wenhan Lin ${ }^{5, *}$ and Peter Proksch ${ }^{1, *}$

1 Institute of Pharmaceutical Biology and Biotechnology, Heinrich-Heine University, Universitaetsstrasse 1, D-40225 Duesseldorf, Germany; E-Mail: sherif.Elsayed@uni-duesseldorf.de (S.S.E.)

2 Helmholtz Center for Infection Research, Inhoffenstrasse 7, D-38124 Braunschweig, Germany; E-Mail: victor.wray@helmholtz-hzi.de (V.W.)

3 National Museum of Natural History, PO Box 95172300 RA Leiden, The Netherlands; E-Mail: voogd@naturalis.nnm.nl (N.J.V.)

4 Analytical and Testing Center, Beijing Normal University, Beijing 100875, China; E-Mail: dengzw@bnu.edu.cn (Z.W.D.)

5 State Key Laboratory of Natural and Biomimetic Drugs, Peking University, Beijing 100083, China

6 Department of Pharmacognosy and Phytochemistry, Faculty of Pharmacy, Ain-Shams University, Abbasia, Cairo, Egypt; E-Mail: sss3ebada@yahoo.com (S.S.E.)

* Authors to whom correspondence should be addressed; E-Mail: proksch@uni-duesseldorf.de (P.P.); Tel.: +49211-81-14163; Fax: +49211-81-11923; or E-Mail: whlin@bjmu.edu.cn (W.H.L.); Tel.: +8610-82806188; Fax: $+8610-82802724$.

Received: 21 August 2009; in revised form: 2 September 2009 / Accepted: 14 September 2009 / Published: 15 September 2009

\begin{abstract}
Two new jaspamide derivatives $\mathbf{2}$ and 3, together with the parent compound jaspamide (1) have been isolated from the marine sponge Jaspis splendens collected in Kalimantan (Indonesia). The structures of the new compounds were unambiguously elucidated based on 1D and 2D NMR spectral data, mass spectrometry and comparison with jaspamide (1). The new derivatives inhibited the growth of mouse lymphoma (L5178Y) cell line in vitro with $\mathrm{IC}_{50}$ values of $<0.1 \mu \mathrm{g} / \mathrm{mL}$.
\end{abstract}

Keywords: Jaspis splendens; jaspamide Q and R; structure elucidation; cytotoxic activity 


\section{Introduction}

Peptides are well-known bioactive metabolites from marine invertebrates [1,2] including linear peptides [3,4], depsipeptides [5,6], cyclic [7-11] and bicyclic peptides [12,13]. Sponges of the genus Jaspis (family, Jaspidae) have been a rich source of biologically active, structurally novel natural products. After the discovery of the cyclodepsipeptide jaspamide (jasplakinolide, 1) in the sponge Jaspis cf. johnstoni in 1986 by Ireland [14] and Crews [15], which is known for its pronounced biological activities including antifungal [16], anthelmintic, insecticidal [14,15] and cytotoxic activity [17], sponges of the genus Jaspis have received considerable attention, and since then the chemistry of Jaspis sponges has been the subject of more than 90 publications. A wide variety of constituents has been isolated from this genus including several jaspamide derivatives from Jaspis splendens [18-21], isomalabaricane triterpenes from Jaspis stellifera [22-24] and other species [25-29], cytotoxic macrolides from the Okinawan sponge Jaspis sp. [30], bengazoles [31,32] that stand out as unique bis-oxazoles containing a carbohydrate-like polyol side chain, antiparasitic, antimicrobial, and cytotoxic amino acid derivatives known as bengamides [33-35], cytotoxic bromotyrosine derivatives [36,37], and a series of dihydroxystyrene sulphate derivatives [38-42].

Figure 1. New jaspamide derivatives $\mathbf{2}$ and 3, together with the parent jaspamide (1).

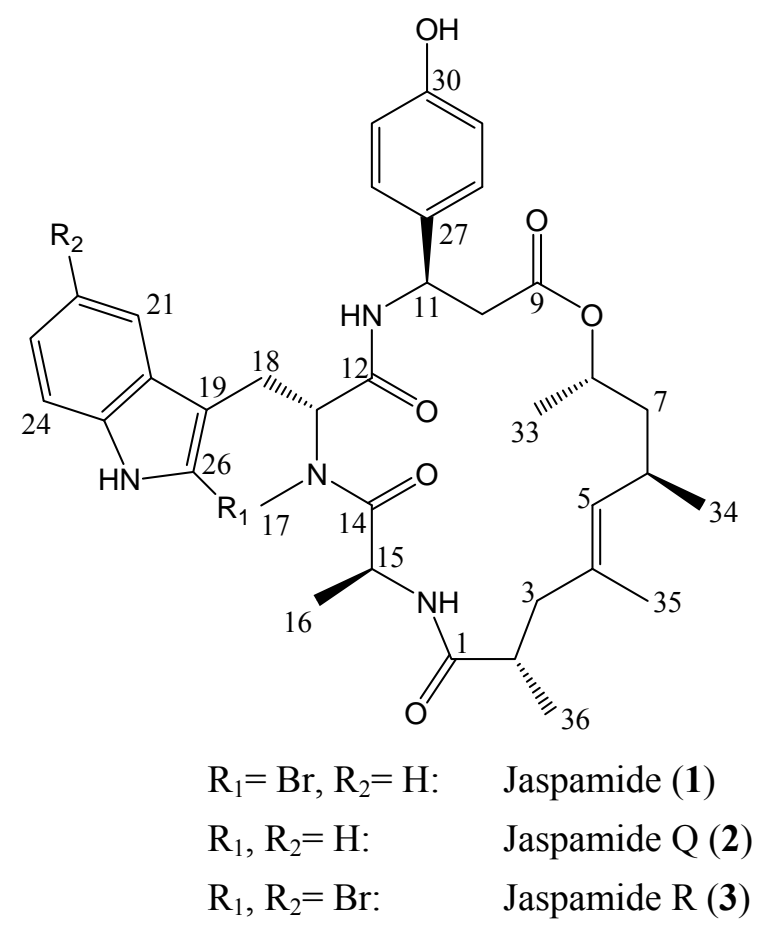

As part of our ongoing studies on bioactive natural products from marine sponges, we investigated a specimen of Jaspis splendens collected in Kalimantan (Indonesia). The crude methanolic extract exhibited considerable in vitro cytotoxic activity against mouse lymphoma L5178Y cells. Chromatographic separation of the extract yielded jaspamide (1) as the major constituent. Through bioactivity-guided chemical investigation of the ethyl acetate soluble fraction minor analogues of jaspamide, including the new natural products jaspamide Q (2), and jaspamide R (3) (Figure 1) were obtained. In this paper, we describe isolation, structural elucidation, and biological activity of the new 
jaspamide derivatives, both of which carry a modified 2-bromoabrine ( $N$-methyltryptophan) residue compared to jaspamide (1).

\section{Results and Discussion}

Total methanolic extract of the sponge J. splendens was partitioned according to the scheme previously described by Ebada et al. [43]. The bioactive ethyl acetate soluble fraction was further chromatographed by Sephadex LH20 column chromatography followed by reversed-phase preparative HPLC to give jaspamide (1, 0.0013\%, dry weight), jaspamide Q (2, 0.00001\%, dry weight), and jaspamide R (3,0.00001\%, dry weight).

Jaspamide Q (2) was obtained as a white amorphous solid, and the ESIMS spectrum showed a pseudomolecular ion peak at $\mathrm{m} / \mathrm{z} 631.3[\mathrm{M}+\mathrm{H}]^{+}$, which was 79 amu smaller than that of jaspamide (1), the parent compound. This difference was assigned to the absence of the bromine atom at C-26 in jaspamide (1). The molecular formula of jaspamide Q (2) was $\mathrm{C}_{36} \mathrm{H}_{46} \mathrm{~N}_{4} \mathrm{O}_{6}$, based on HRFTMS $\left(\mathrm{m} / \mathrm{z} 631.3491[\mathrm{M}+\mathrm{H}]^{+}, \Delta+1.0 \mathrm{ppm}\right)$, therefore jaspamide $\mathrm{Q}(\mathbf{2})$ was identified as the debromo analogue of $1 .{ }^{1} \mathrm{H}-\mathrm{NMR}$ spectral data (Table 1) revealed that the resonances of 2 were superimposable with those of 1 with only one additional proton resonance at $\delta_{\mathrm{H}} 6.87(1 \mathrm{H}, \mathrm{br} s)$ that was ascribed to $\mathrm{H}-26$. The complete structure of jaspamide Q (2) was unambiguously elucidated and assigned on the basis of ${ }^{1} \mathrm{H}-{ }^{1} \mathrm{H}$ COSY, TOCSY, ROESY, and HMBC spectra.

In particular, the similarity of ${ }^{1} \mathrm{H}$-, and ${ }^{13} \mathrm{C}-\mathrm{NMR}$ resonances between jaspamide $\mathrm{Q}$ (2) and jaspamide (1) implied that the chiral centers of alanine, abrine ( $N$-methyltryptophan), $\beta$-tyrosine, and of the polypropionate fragment had the same relative configurations in both molecules. Therefore, the stereochemistry depicted in Figure 1 was tentatively assigned by analogy with the parent compound together with ROESY spectra that revealed a clear correlation between Me-16 and Me-34; and Me-33 and Me-36. However, an apparent deshielding of Me-16 was noted in 2 [ $\delta_{\mathrm{H}} 1.04(3 \mathrm{H}, d, 6.6 \mathrm{~Hz})$ ] compared to $1\left[\delta_{\mathrm{H}} 0.70(3 \mathrm{H}, d, 6.7 \mathrm{~Hz})\right]$ resembling that between jaspamide $\mathrm{M}\left[\delta_{\mathrm{H}} 1.17(3 \mathrm{H}, d\right.$, $6.8 \mathrm{~Hz})][21]$ and jaspamide $\mathrm{H}\left[\delta_{\mathrm{H}} 0.72(3 \mathrm{H}, d, 6.7 \mathrm{~Hz})\right]$ [20]. These differences in chemical shifts for the latter congeners were proven to be caused by D-Ala or L-Ala residues, respectively. Anaylsis of the absolute configurations of the amino acids of 2 could not be performed due to the small amount of compound isolated $(0.7 \mathrm{mg})$.

A partial ${ }^{13} \mathrm{C}-\mathrm{NMR}$ assignment of jaspamide Q (2) was achieved through HMBC spectra (Figure 2) which revealed clear correlations at $\delta_{\mathrm{c}} 174.5, \delta_{\mathrm{c}} 40.4, \delta_{\mathrm{c}} 40.9, \delta_{\mathrm{c}} 133.6, \delta_{\mathrm{c}} 128.2, \delta_{\mathrm{c}} 29.5, \delta_{\mathrm{c}} 43.6$, $\delta_{\mathrm{c}} 70.4, \delta_{\mathrm{c}} 56.4, \delta_{\mathrm{c}} 173.6$, and $\delta_{\mathrm{c}} 46.0 \mathrm{ppm}$ that were ascribed to $\mathrm{C}-1$ to $\mathrm{C}-8$, and $\mathrm{C}-13$ to $\mathrm{C}-15$, respectively. Moreover, $\mathrm{HMBC}$ spectra evidenced and confirmed the amino acid sequence in jaspamide Q (2) through cross-peaks between NH-Tyr and C-12, Me-17 and C-14, and between $\mathrm{NH}$-Ala and C-1.

Jaspamide R (3) was isolated as a white amorphous solid. Its ESI mass spectrum exhibited pseudomolecular ion peaks at $\mathrm{m} / \mathrm{z} 787.1,789.1$, and $791.1[\mathrm{M}+\mathrm{H}]^{+}$, in a ratio of $1: 2: 1$, supporting the existence of two bromine atoms in the compound. The molecular formula of jaspamide $\mathrm{R}$ (3) was determined to be $\mathrm{C}_{36} \mathrm{H}_{44} \mathrm{Br}_{2} \mathrm{~N}_{4} \mathrm{O}_{6}$ by HRFTMS (m/z 789.1691 [M+H] $\left.]^{+}, \Delta+1.0 \mathrm{ppm}\right)$ which exceeds that of jaspamide (1) by 79 amu revealing that jaspamide $R$ (3) is a dibromo analogue of jaspamide Q (2). 
Figure 2. Key HMBC correlations of jaspamide Q (2).

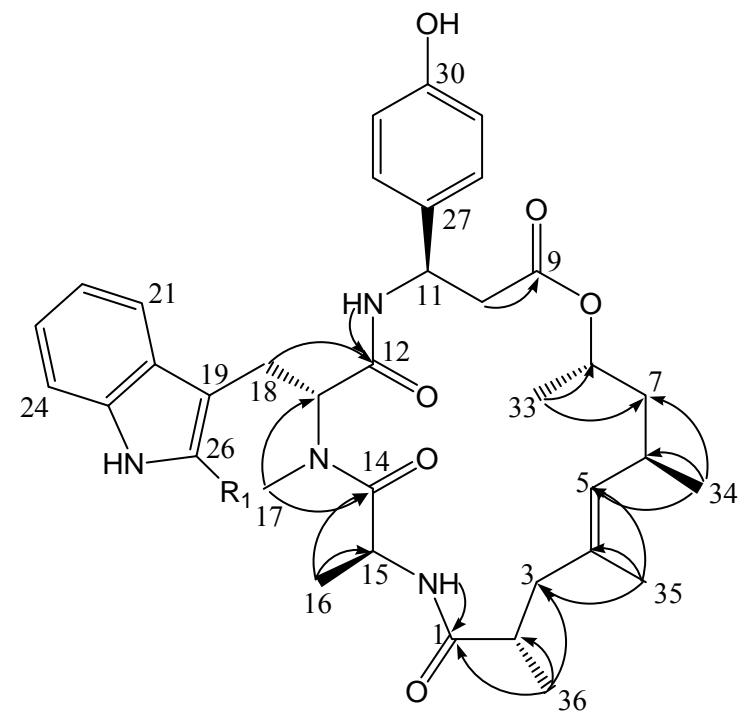

This difference was explained by the ${ }^{1} \mathrm{H}-\mathrm{NMR}$ spectral data (Table 1), which revealed close similarity between jaspamide R (3) and jaspamide (1), except for the proton resonances corresponding to the indole moiety of the 2-bromoabrine unit. Jaspamide $\mathrm{R}(\mathbf{3})$ showed three proton resonances at $\delta_{\mathrm{H}} 7.41(1 \mathrm{H}$, br $s), \delta_{\mathrm{H}} 7.20(1 \mathrm{H}, d, 8.2 \mathrm{~Hz})$, and $\delta_{\mathrm{H}} 7.42(1 \mathrm{H}, d, 8.2 \mathrm{~Hz})$ that were assigned to $\mathrm{H}-21$, $\mathrm{H}-23$, and $\mathrm{H}-24$, respectively. Whereas for jaspamide (1), four resonances, at $\delta_{\mathrm{H}} 7.23(1 \mathrm{H}, d, 8.0 \mathrm{~Hz})$, $\delta_{\mathrm{H}} 7.10(1 \mathrm{H}, t, 8.0 \mathrm{~Hz}), \delta_{\mathrm{H}} 7.12(1 \mathrm{H}, t, 8.0 \mathrm{~Hz})$, and $\delta_{\mathrm{H}} 7.54(1 \mathrm{H}, d, 8.0 \mathrm{~Hz})$, ascribed for $\mathrm{H}-21$ to H-24, were observed. Based on this finding, the additional bromine atom of $\mathbf{3}$ was assumed to be located at $\mathrm{C}-22$. This hypothesis was further confirmed by $2 \mathrm{D}$ NMR spectral analyses including ${ }^{1} \mathrm{H}-{ }^{1} \mathrm{H}$ COSY, TOCSY, and NOESY spectra that revealed a clear NOE correlation between proton resonance at $\delta_{\mathrm{H}} 7.41(1 \mathrm{H}$, br $s)$, and $\mathrm{NH}$-Tyr at $\delta_{\mathrm{H}} 7.53(1 \mathrm{H}, d, 8.6 \mathrm{~Hz})$ proving the attachment of the second bromine atom to be at $\mathrm{C}-22$. Moreover, the close resemblance in ${ }^{1} \mathrm{H}$ resonances between jaspamide $\mathrm{R}$ (3), and jaspamide (1) supports the notion that the chiral centers of alanine, 2,4-dibromoabrine, B-tyrosine, and the polypropionate fragment have the same relative configurations in both molecules (Figure 1). Again, due to the lack of material isolated of $3(0.5 \mathrm{mg})$, analysis of the absolute configurations of the amino acids, e.g. by Marfey's method, could not be performed.

To the best of our knowledge, fifteen jaspamide congeners (B-P) were hitherto isolated from the genus Jaspis [14,18-21] and all of them show antiproliferative activity with $\mathrm{IC}_{50}$ values ranging from 0.01 to $33 \mu \mathrm{M}$ against human breast adenocarinoma (MCF-7), and colon carcinoma (H-29) cell lines [21].

Jaspamide Q (2) and R (3) together with the parent jaspamide (1) differ in the bromination pattern of the abrine ( $N$-methyltryptophan) moiety. Since these modifications were claimed as essential for the observed biological activity [44], compounds ( 2 and $\mathbf{3}$ ) together with jaspamide (1) were subjected to a cytotoxicity (MTT) assay against mouse lymphoma (L5178Y) cell lines. They exhibited potent activities with $\mathrm{IC}_{50}$ values in the $\mathrm{ng} / \mathrm{mL}$ range $(<0.1 \mu \mathrm{g} / \mathrm{mL},<0.16 \mu \mathrm{M})$, compared to kahalalide $\mathrm{F}$ $\left(\mathrm{IC}_{50}=6.3 \mu \mathrm{g} / \mathrm{mL}, 4.3 \mu \mathrm{M}\right)$ which was used as a positive control. Further studies aimed at determining the effect of bromination pattern of the abrine residue on cytototoxic activity are in progress. 
Table 1. ${ }^{1} \mathrm{H}-\mathrm{NMR}$ data of jaspamide Q (2), and $\mathrm{R}(\mathbf{3}),\left(500 \mathrm{MHz}, \mathrm{CDCl}_{3}\right.$-d).

\begin{tabular}{|c|c|c|}
\hline \multirow{2}{*}{ Position } & \multirow{2}{*}{$\begin{array}{c}\text { Jaspamide Q (2) } \\
\delta_{\mathrm{H}} \text { mult. }(\mathrm{J} \text { Hz) }\end{array}$} & \multirow{2}{*}{$\begin{array}{c}\text { Jaspamide R (3) } \\
\delta_{H} \text { mult. }(J \text { Hz) }\end{array}$} \\
\hline & & \\
\hline 2 & $2.51(1 \mathrm{H}, m)$ & $2.49(1 \mathrm{H}, m)$ \\
\hline \multirow[t]{2}{*}{3} & A) $2.37(1 \mathrm{H}, d d, 11.5,15.8 \mathrm{~Hz})$ & A) $2.37(1 \mathrm{H}, \mathrm{dd}, 11.5,15.8 \mathrm{~Hz})$ \\
\hline & B) $1.90(1 \mathrm{H}, d, 15.8 \mathrm{~Hz})$ & B) $1.90(1 \mathrm{H} . d, 15.8 \mathrm{~Hz})$ \\
\hline 5 & $4.79(1 \mathrm{H}, d, 6.7 \mathrm{~Hz})$ & $4.74(1 \mathrm{H}, d, 6.7 \mathrm{~Hz})$ \\
\hline 6 & $2.23(1 \mathrm{H}, m)$ & $2.23(1 \mathrm{H}, m)$ \\
\hline 7 & A) $1.32(1 \mathrm{H}, m)$, B) $1.10(1 \mathrm{H}, m)$ & A) $1.32(1 \mathrm{H}, m)$, B) $1.10(1 \mathrm{H}, m)$ \\
\hline 8 & $4.64(1 \mathrm{H}, m)$ & $4.60(1 \mathrm{H}, m)$ \\
\hline \multirow[t]{2}{*}{10} & A) $2.67(1 \mathrm{H}, d d, 4.7,15.0 \mathrm{~Hz})$ & A) $2.67(1 \mathrm{H}, d d, 4.7,15.0 \mathrm{~Hz})$ \\
\hline & B) $2.61(1 \mathrm{H}, d d, 5.7,14.8 \mathrm{~Hz})$ & B) $2.61(1 \mathrm{H}, d d, 5.7,14.8 \mathrm{~Hz})$ \\
\hline 11 & $5.25(1 \mathrm{H}, d d, 5.3,8.6 \mathrm{~Hz})$ & $5.25(1 \mathrm{H}, d d, 5.3,8.6 \mathrm{~Hz})$ \\
\hline 13 & $5.63(1 \mathrm{H}, d d, 6.3,10.0 \mathrm{~Hz})$ & $5.73(1 \mathrm{H}, d d, 6.3,10.0 \mathrm{~Hz})$ \\
\hline 15 & $4.77(1 \mathrm{H}, m)$ & $4.72(1 \mathrm{H}, m)$ \\
\hline 16 & $1.04(3 \mathrm{H}, d, 6.6 \mathrm{~Hz})$ & $0.81(3 \mathrm{H}, d, 6.7 \mathrm{~Hz})$ \\
\hline 17 & $2.97(3 \mathrm{H}, \mathrm{s})$ & $2.98(3 \mathrm{H}, \mathrm{s})$ \\
\hline \multirow[t]{2}{*}{18} & A) $3.43(1 \mathrm{H}, d d, 6.3,15.5 \mathrm{~Hz})$ & A) $3.33(1 \mathrm{H}, d d, 6.3,15.5 \mathrm{~Hz})$ \\
\hline & B) $3.17(1 \mathrm{H}, d d, 10.4,15.2 \mathrm{~Hz})$ & B) $3.18(1 \mathrm{H}, d d, 10.4,15.2 \mathrm{~Hz})$ \\
\hline 21 & $7.61(1 \mathrm{H}, d, 8.0 \mathrm{~Hz})$ & $7.41(1 \mathrm{H}$, br s) \\
\hline 22 & $7.11(1 \mathrm{H}, t, 8.0 \mathrm{~Hz})$ & \\
\hline 23 & $7.18(1 \mathrm{H}, t, 8.0 \mathrm{~Hz})$ & $7.20(1 \mathrm{H}, d, 8.2 \mathrm{~Hz})$ \\
\hline 24 & $7.35(1 \mathrm{H}, d, 8.0 \mathrm{~Hz})$ & $7.42(1 \mathrm{H}, d, 8.2 \mathrm{~Hz})$ \\
\hline 26 & $6.87(1 \mathrm{H}$, br $s)$ & \\
\hline 28 & $6.90(1 \mathrm{H}, d, 8.3 \mathrm{~Hz})$ & $6.98(1 \mathrm{H}, d, 8.4 \mathrm{~Hz})$ \\
\hline 29 & $6.69(1 \mathrm{H}, d, 8.3 \mathrm{~Hz})$ & $6.71(1 \mathrm{H}, d, 8.4 \mathrm{~Hz})$ \\
\hline 31 & $6.69(1 \mathrm{H}, d, 8.3 \mathrm{~Hz})$ & $6.71(1 \mathrm{H}, d, 8.4 \mathrm{~Hz})$ \\
\hline 32 & $6.90(1 \mathrm{H}, d, 8.3 \mathrm{~Hz})$ & $6.98(1 \mathrm{H}, d, 8.4 \mathrm{~Hz})$ \\
\hline 33 & $1.06(3 \mathrm{H}, d, 6.3 \mathrm{~Hz})$ & $1.05(3 \mathrm{H}, d, 6.1 \mathrm{~Hz})$ \\
\hline 34 & $0.83(3 \mathrm{H}, d, 6.5 \mathrm{~Hz})$ & $0.81(3 \mathrm{H}, d, 6.7 \mathrm{~Hz})$ \\
\hline 35 & $1.59(3 \mathrm{H}, \mathrm{s})$ & $1.57(3 \mathrm{H}, s)$ \\
\hline 36 & $1.15(3 \mathrm{H}, d, 6.8 \mathrm{~Hz})$ & $1.13(3 \mathrm{H}, d, 6.8 \mathrm{~Hz})$ \\
\hline $\mathrm{NH}$-Tyr & $7.46(1 \mathrm{H}, d, 8.6 \mathrm{~Hz})$ & $7.53(1 \mathrm{H}, d, 8.6 \mathrm{~Hz})$ \\
\hline $\mathrm{NH}$-Trp & $8.24(1 \mathrm{H}$, br $s)$ & $8.42(1 \mathrm{H}$, br $s)$ \\
\hline $\mathrm{NH}$-Ala & $6.73(1 \mathrm{H}, d, 6.4 \mathrm{~Hz})$ & $6.65(1 \mathrm{H}, d, 6.5 \mathrm{~Hz})$ \\
\hline
\end{tabular}

\section{Experimental Section}

General experimental procedures

Column chromatography was carried out on Sephadex LH-20 using methanol as an eluent. For analytical HPLC analysis, samples were injected into a HPLC system equipped with a photodiode array detector (Dionex, Munich, Germany). Routine detection was at 235, 254, 280, and $340 \mathrm{~nm}$. The separation column $(125 \times 4 \mathrm{~mm}$ ID) was prefilled with C-18 Eurosphere, $5 \mu \mathrm{m}$ (Knauer, Berlin, Germany). Separation was achieved by applying a linear gradient from $90 \% \mathrm{H}_{2} \mathrm{O}(\mathrm{pH} 2.0)$ to 
$100 \% \mathrm{MeOH}$ over $40 \mathrm{~min}$. TLC analysis was carried out using aluminium sheet precoated with silica gel $60 \mathrm{~F}_{254}$ (Merck, Darmstadt, Germany).

Preparative HPLC separations were performed on a LaChrom-Merck Hitachi HPLC system, pump L-7100, UV detector L-7400 using a C-18 column (Knauer, $300 \times 8 \mathrm{~mm}$ ID, prefilled with C-18 Eurosphere, flow rate $5 \mathrm{~mL} / \mathrm{min}$, UV detection at $280 \mathrm{~nm}$ ), and the solvent system consisted of a linear gradient of $\mathrm{MeOH}$ and nanopure $\mathrm{H}_{2} \mathrm{O}$.

Optical rotations were measured on a Perkin-Elmer-241 MC polarimeter. ESIMS were obtained on a ThermoFinnigan LCQ DECA mass spectrometer coupled to an Agilent 1100 HPLC system equipped with a photodiode array detector. HRFTMS was recorded on a LTQ FT-MS-Orbitrap (ThermoFinnigan, Bremen, Germany). 1D and 2D NMR spectra were recorded at $300^{\circ} \mathrm{K}$ on a Bruker ARX-500. Samples were dissolved in deuterochloroform.

\section{Biological material}

In August 2008, specimens of $J$. splendens were collected on three neighboring Islands from East Kalimantan (Indonesia), namely Samama, Panjang, and Shoal Islands, at 10 meter depths. Numbers of voucher specimens are RMNH Por. 4234, 4266 and 4299, respectively. They were taxonomically identified as Jaspis splendens (order Astrophorida, family Ancorinidae) at the National Museum of Natural History, Leiden, Netherlands. HPLC and LCMS analyses of the three samples revealed that they were identical with regard to their peptide derivatives. Hence, the material was combined in order to obtain sufficient amounts of compounds for subsequent structure elucidation.

\section{Extraction and isolation}

The animal was freeze-dried, and the material $(500 \mathrm{~g})$ was extracted with methanol $(3 \times 2 \mathrm{~L})$ and filtered. The extract was then combined, evaporated to dryness, and partitioned as follows. The methanolic extract ( $80 \mathrm{~g}$ ) was dissolved in water and partitioned against $n$-hexane, ethyl acetate, and then $n$-butanol. The bioactive ethyl acetate soluble fraction $(2 \mathrm{~g})$ was chromatographed by CC using Sephadex LH20 as stationary phase and eluted with methanol followed by reversed-phase (C18 Eurosphere 100) HPLC using gradient elution of $\mathrm{MeOH}: \mathrm{H}_{2} \mathrm{O}$ to yield $105 \mathrm{mg}$ of jaspamide (1), $0.7 \mathrm{mg}$ of jaspamide Q (2), and $0.5 \mathrm{mg}$ of jaspamide R (3).

Jaspamide Q (2) was obtained as a white amorphous solid: $[\alpha]_{\mathrm{D}}^{20}-62.0^{\circ}\left(c 0.01, \mathrm{CHCl}_{3}\right) ; \mathrm{UV}(\mathrm{MeOH})$ $\lambda_{\max } 226,280 \mathrm{~nm} ;{ }^{1} \mathrm{H}-\mathrm{NMR}$ see Table 1; ESI-MS pos $\mathrm{m} / \mathrm{z} 631.3[\mathrm{M}+\mathrm{H}]^{+}$(100), ESI-MS neg m/z 629.3 [M-H] $]^{-}$(100), HRFTMS m/z $631.3491[\mathrm{M}+\mathrm{H}]^{+}$(calcd for $\mathrm{C}_{36} \mathrm{H}_{47} \mathrm{~N}_{4} \mathrm{O}_{6}, 631.3490$ ), and $\mathrm{m} / \mathrm{z} 653.3307$ $[\mathrm{M}+\mathrm{Na}]^{+}$(calcd for $\mathrm{C}_{36} \mathrm{H}_{46} \mathrm{~N}_{4} \mathrm{O}_{6} \mathrm{Na}, 653.3310$ ).

Jaspamide $R(3)$ was obtained as a white amorphous solid: $[\alpha]_{\mathrm{D}}^{20}-100.0^{\circ}\left(c \quad 0.01, \mathrm{CHCl}_{3}\right) ; \mathrm{UV}(\mathrm{MeOH})$ $\lambda_{\max }$ 231, $283 \mathrm{~nm} ;{ }^{1} \mathrm{H}-\mathrm{NMR}$ see Table 1; ESI-MS pos $\mathrm{m} / \mathrm{z}$ 787.1, 789.1, $791.1[\mathrm{M}+\mathrm{H}]^{+}, 1: 2: 1$; ESI-MS neg $\mathrm{m} / \mathrm{z}$ 785.2, 787.1, $789.0 \quad[\mathrm{M}-\mathrm{H}]^{-}, \quad 1: 2: 1 ;$ HRFTMS $\mathrm{m} / \mathrm{z} 789.1691[\mathrm{M}+\mathrm{H}]^{+}$(calcd for $\mathrm{C}_{36} \mathrm{H}_{45} \mathrm{~N}_{4} \mathrm{O}_{6}{ }^{79} \mathrm{Br}_{2}, 789.1680$ ), and $\mathrm{m} / \mathrm{z} 811.1507[\mathrm{M}+\mathrm{Na}]^{+}$(calcd for $\mathrm{C}_{36} \mathrm{H}_{44} \mathrm{~N}_{4} \mathrm{O}_{6}{ }^{79} \mathrm{Br}_{2} \mathrm{Na}, 811.1499$ ). 


\section{Cell proliferation assay}

Cytotoxicity was tested against L5178Y mouse lymphoma cells using the microculture tetrazolium (MTT) assay as described earlier [11,45]. All experiments were carried out in triplicate and repeated three times. As controls, media with 0.1\% EGMME/DMSO were included in the experiments.

\section{Acknowledgements}

A scholarship granted and financed by the Egyptian Government (Ministry of High Education) to S.S.E. is gratefully acknowledged. This project was supported by a grant of BMBF (to P.P.) and MOST (to W.L.). We are indebted to Prof. Dr. W.E.G. Mueller (University of Mainz) for performing the cytotoxicity (MTT) assay.

\section{References and Notes}

1. Blunt, J.W.; Copp, B.R.; Hu, W.-P.; Munro, M.H.G.; Northcote, P.T.; Prinsep, M.R. Marine natural products. Nat. Prod. Rep. 2009, 26, 170-244.

2. Hamann, M.T.; Scheuer, P.J. Kahalalide F: A bioactive depsipeptide from the sacoglossan mollusk Elysia rufescens and the green alga Bryopsis sp. J. Am. Chem. Soc. 1993, 115, 5825-5826.

3. Hamada, T.; Matsunaga, S.; Yano, G.; Fusetani, N. Polytheonamides A and B, highly cytotoxic, linear polypeptides with unprecedented structural features, from the marine sponge, Theonella swinhoei. J. Am. Chem. Soc. 2005, 127, 110-118.

4. Nakao, Y.; Masuda, A.; Matsunaga, S.; Fusetani, N. Pseudotheonamides, serine protease inhibitors from the marine sponge Theonella swinhoei. J. Am. Chem. Soc. 1999, 121, 2425-2431.

5. Ford, P.W.; Gustafson, K.R.; McKee, T.C.; Shigematsu, N.; Maurizi, L.K.; Pannell, L.K.; Williams, D.E.; Dilip de Silva, E.; Lassota, P.; Allen, T.M.; van Soest, R.; Andersen, R.J.; Boyd, M.R. Papuamides A-D, HIV-inhibitory and cytotoxic depsipeptides from the sponges Theonella mirabilis and Theonella swinhoei collected in Papua New Guinea. J. Am. Chem. Soc. 1999, 121, 5899-5909.

6. Reese, M.T.; Gulavita, N.K.; Nakao, Y.; Hamann, M.T.; Yoshida, W.Y.; Coval, S.J.; Scheuer, P.J. Kulolide: A cytotoxic depsipeptide from a cephalaspidean mollusk, Philinopsis speciosa. J. Am. Chem. Soc. 1996, 118, 11081-11084.

7. Renner, M.K.; Shen, Y.C.; Cheng, X.C.; Jensen, P.R.; Frankmoelle, W.; Kauffman, C.A.; Fenical, W.; Emil, C.J. Cyclomarins A-C, new anti-inflammatory cyclic peptides produced by a marine bacterium Streptomyces sp. J. Am. Chem. Soc. 1999, 121, 11273-11276.

8. Clark, W.D.; Corbett, T.; Valeriote, F.; Crews, P. Cylcocinamide A, an unusual cytotoxic halogenated hexapeptide from the marine sponge Psammocinia. J. Am. Chem. Soc. 1997, 119, 9285-9286.

9. Nakao, Y.; Yeung, B.K.S.; Yoshida, W.Y.; Scheuer, P.J.; Kelly-Borges, M. Kapakahine B: A cyclic hexapeptide with an $\alpha$-carboline ring system from the marine sponge Cribrochalina olemda. J. Am. Chem. Soc. 1995, 117, 8271-8272. 
10. Fusetani, N.; Sugawara, T.; Matsunaga, S.; Hirota, H. Orbiculamide A: A novel cytotoxic cyclic peptide from a marine sponge Theonella sp. J. Am. Chem. Soc. 1991, 113, 7811-7812.

11. Ashour, M.; Edrada, R.A.; Ebel, R.; Wray, V.; Waetjen, W.; Padmakumar, K.; Mueller, W.E.G.; Lin W.H.; Proksch, P. Kahalalide derivatives from the Indian sacoglossan mollusk Elysia grandifolia. J. Nat. Prod. 2006, 69, 1547-1553.

12. Matsunaga, S.; Fusetani, N.; Hashimoto, K.; Walchli, M. Theonellamide F: A novel antifungal bicyclic peptide from a marine sponge Theonella sp. J. Am. Chem. Soc. 1989, 111, 2582-2588.

13. Ireland, C.; Scheuer, P.J. Ulicyclamide and ulithiacyclamide, two new small peptides from a marine tunicate. J. Am. Chem. Soc. 1980, 102, 5688-5691.

14. Zabriskie, T.E.; Klocke, J.A.; Ireland, C.M.; Marcus, A.H.; Molinski, T.F.; Faulkner, D.J.; Xu, C.; Clardy, J.C. Jaspamide, a modified peptide from a Jaspis sponge, with insecticidal and antifungal activity. J. Am. Chem. Soc. 1986, 108, 3123-3124.

15. Crews, P.; Manes, L.V.; Boehler, M. Jasplakinolide, a cyclodepsipeptide from the marine sponge, Jaspis sp. Tetrahedron Lett. 1986, 27, 2797-2800.

16. Scott, V.R.; Boehme, R.; Matthews, T.R. New class of antifungal agents: Jasplakinolide, a cyclodepsipeptide from the marine sponge, Jaspis species. Antimicrob. Agents Chemother. 1988, 32, 1154-1157.

17. Inman, W.; Crews, P. Novel marine sponge derived amino acids, 8. conformational analysis of jasplakinolide. J. Am. Chem. Soc. 1989, 111, 2822-2829.

18. Zampella, A.; Giannini, C.; Debitus, C.; Roussakis, C.; D’Auria, M.V. New jaspamide derivatives from the marine sponge Jaspis splendans collected in Vanuatu. J. Nat. Prod. 1999, 62, 332-334.

19. Gala, F.; D’Auria, M.V.; De Marino, S.; Zollo, F.; Smith, C.D.; Copper, J.E.; Zampella, A. New jaspamide derivatives with antimicrofilament activity from the sponge Jaspis splendans. Tetrahedron 2007, 63, 5212-5219.

20. Gala, F.; D’Auria, M.V.; De Marino, S.; Sepe, V.; Zollo, F.; Smith, C.D.; Copper, J.E.; Zampella, A. Jaspamides $\mathrm{H}-\mathrm{L}$, new actin-targeting depsipeptides from the sponge Jaspis splendans. Tetrahedron 2008, 64, 7127-7130.

21. Gala, F.; D’Auria, M.V.; De Marino, S.; Sepe, V.; Zollo, F.; Smith, C.D.; Keller, S.N.; Zampella, A. Jaspamides M-P: New tryptophan modified jaspamide derivatives from the sponge Jaspis splendans. Tetrahedron 2009, 65, 51-56.

22. Ravi, B.N.; Wells, R.J.; Croft, K.D. Malabaricane triterpenes from a Fijian collection of the sponge Jaspis stellifera. J. Org. Chem. 1981, 46, 1998-2001.

23. Tsuda, M.; Ishibashi, M.; Agemi, K.; Sasaki, T.; Kobayashi, J. Stelliferins A-F, new antineoplastic isomalabaricane triterpenes from the Okinawan marine sponge Jaspis stellifera. Tetrahedron 1991, 47, 2181-2194.

24. Kobayashi, J.; Yuasa, K.; Kobayashi, T.; Sasaki, T.; Tsuda, M. Jaspiferals A-G, new cytotoxic isomalabaricane-type nortriterpenes from Okinawan marine sponge Jaspis stellifera. Tetrahedron 1996, 52, 5745-5750.

25. Zampella, A.; D'Auria, M.V.; Debitus, C.; Menou, J.-L. New isomalabaricane derivatives from a new species of Jaspis sponge collected at the Vanuatu islands. J. Nat. Prod. 2000, 63, 943-946.

26. Meragelman, K.M.; McKee, T.C.; Boyd, M.R. New cytotoxic isomalabaricane triterpenes from the sponge Jaspis species. J. Nat. Prod. 2001, 64, 389-392. 
27. Tang, S.A.; Deng, Z.W.; Li, J.; Fu, H.Z.; Pei, Y.H.; Zhang, S.; Lin, W.H. A new isomalabaricane triterpenoid from sponge Jaspis sp. Chin. Chem. Lett. 2005, 16, 353-355.

28. Tang, S.; Pei, Y.; Fu, H.; Deng, Z.; Li, J.; Proksch, P.; Lin, W. Jaspolides A-F, six new isomalabaricane-type terpenoids from the sponge Jaspis sp. Chem. Pharm. Bull. 2006, 54, 4-8.

29. Tang, S.; Deng, Z.; Proksch, P.; Lin, W. Jaspolides G and H, unique bisisomalabaricane from the Chinese marine sponge Jaspis sp. Tetrahedron Lett. 2007, 48, 5443-5447.

30. Kobayashi, J.; Murata, O.; Shigemori, H.; Sasaki, T. Jaspisamides A-C, new cytotoxic macrolides from the Okinawan marine sponge Jaspis sp. J. Nat. Prod. 1993, 56, 787-791.

31. Rodriguez, J.; Nieto, R.M.; Crews, P. New structures and bioactivity patterns of bengazole alkaloids from a choristid marine sponge. J. Nat. Prod. 1993, 56, 2034-2040.

32. Searle, P.A.; Richter, R.K.; Molinski, T.F. Bengazoles $\mathrm{C}-\mathrm{G}$ from the sponge Jaspis sp. synthesis of the side chain and determination of absolute configuration. J. Org. Chem. 1996, 61, 40734079.

33. D’Auria, M.V.; Giannini, C.; Minale, L.; Zampella, A.; Debitus, C.; Frostin, M. Bengamides and related amino acid derivatives from the new Caledonian marine sponge Jaspis carteri. J. Nat. Prod. 1997, 60, 814-816.

34. Groweiss, A.; Newcomer, J.J.; O’Keefe, B.R.; Blackman, A.; Boyd, M.R. Cytotoxic metabolites from an Australian collection of the sponge Jaspis species. J. Nat. Prod. 1999, 62, 1691-1693.

35. Thale, Z.; Kinder, F.R.; Bair, K.W.; Bontempo, J.; Czuchta, A.M.; Versace, R.W.; Philips, P.E.; Sanders, M.L.; Wattanasin, S.; Crews, P. Bengamides revisited: New structures and antitumor studies. J. Org. Chem. 2001, 66, 1733-1741.

36. Park, Y.; Liu, Y.; Hong, J.; Lee, C.-O.; Cho, H.; Kim, D.-K.; Im, K.S.; Jung, J.H. New bromotyrosine derivatives from an association of two sponges, Jaspis wondoensis and Poecillastra wondoensis. J. Nat. Prod. 2003, 66, 1495-1498.

37. Shinde, P.B.; Lee, Y.M.; Dang, H.T.; Hong, J.; Lee, C.-O.; Jung, J.H. Cytotoxic bromotyrosine derivatives from a two-sponge association of Jaspis sp. and Poecillastra sp. Bioorg. Med. Chem. Lett. 2008, 18, 6414-6418.

38. Ohta, S.; Kobayashi, H.; Ikegami, S. Isojaspisin: A novel styryl sulfate from a marine sponge, Jaspis sp., that inhibits hatching of sea urchin embryos. Tetrahedron Lett. 1994, 35, 4579-4580.

39. Tsukamoto, S.; Kato, H.; Hirota, H.; Fusetani, N. Narains: N,N-Dimethylguanidinium styryl sulfates, metamorphosis inducers of ascidian larvae from a marine sponge Jaspis sp. Tetrahedron Lett. 1994, 35, 5873-5874.

40. Ohta, S.; Kobayashi, H.; Ikegami, S. Jaspisin, a novel styryl sulfate from the marine sponge, Jaspis species. Biosci. Biotech. Biochem. 1994, 58, 1752-1753.

41. Tsukamoto, S.; Kato, H.; Hirota, H.; Fusetani, N. 3,4-Dihydroxystyrene dimmers, inducers of larval metamorphosis in ascidians, from a marine sponge Jaspis sp. Tetrahedron 1994, 50, 13583-13592.

42. Chang, Y.H.; Shin, D.; Na, Z.; Lee, H.-S.; Kim, D.-D.; Oh, K.-B.; Shin, J. Dihydroxystyrene metabolites from an association of the sponge Poecillastra wondoensis and Jaspis sp. J. Nat. Prod. 2008, 71, 779-783. 
43. Ebada, S.S.; Edrada, R.A.; Lin, W.; Proksch, P. Methods for isolation, purification and structural elucidation of bioactive secondary metabolites from marine invertebrates. Nat. Prot. 2008, 3, $1820-1831$.

44. Kahn, M.; Nakanishi, H.; Su, T.; Lee, J.H.; Johnson, M. Design and synthesis of nonpeptide mimetics of jaspamide. Int. J. Pept. Protein Res. 1991, 38, 324-334.

45. Carmichael, J.; DeGraff, W.G.; Gazdar, A.F.; Minna, J.D.; Mitchell, J.B. Evaluation of a tetrazolium-based semiautomated colorimetric assay: Assessment of radiosensitivity. Cancer Res. 1987, 47, 943-946.

Samples Availability: Available from the authors.

(C) 2009 by the authors; licensee Molecular Diversity Preservation International, Basel, Switzerland. This article is an open-access article distributed under the terms and conditions of the Creative Commons Attribution license (http://creativecommons.org/licenses/by/3.0/). 